\title{
Morphometric and molecular characterisation of four new Ceratomyxa species (Myxosporea: Bivalvulida: Ceratomyxidae) from fishes off Lizard Island, Australia
}

\author{
Nicole L. Gunter ${ }^{1,2,3}$, Mieke A.A. Burger ${ }^{1,2}$ and Robert D. Adlard ${ }^{1,2}$ \\ ${ }^{1}$ Biodiversity Program, Queensland Museum, P.O. Box 3300, South Brisbane, Queensland 4101, Australia; \\ ${ }^{2}$ School of Chemistry and Molecular Biosciences, The University of Queensland, Brisbane, Queensland 4072, Australia; \\ ${ }^{3}$ Australian National Insect Collection, Commonwealth Scientific and Industrial Research Organisation Entomology, Black Moun- \\ tain Laboratories, P.O. Box 1700, Canberra, ACT 2601, Australia
}

\begin{abstract}
Four new species of Ceratomyxa Thélohan, 1892 are described from the gall bladders of fishes collected off Lizard Island, Australia. These species are characterised using a combination of morphometric and molecular data. Ceratomyxa bartholomewae sp. n. is described from Hyporhamphus dussumieri (Valenciennes) (family Hemirhamphidae); C. koieae sp. n. is described from Sphyraena forsteri Cuvier (family Sphyraenidae); C. pantherini sp. n. is described from Bothus pantherinus (Rüppell) (family Bothidae) and C. reidi sp. n. is described from Chaetodon vagabundus Linnaeus (family Chaetodontidae). A fifth species from Zebrasoma veliferum (Bloch) (family Acanthuridae) is also reported but due to limited material is not formally described here.
\end{abstract}

Keywords: Ceratomyxa, Myxosporea, Myxozoa, phylogeny, SSU rDNA, new species

Ceratomyxa Thélohan, 1892 is the second largest myxozoan genus with just over 260 species described globally. To date, 35 species have been described from the gall bladders of fishes collected from the Great Barrier Reef (GBR) Australia with only 10 of these collected off Lizard Island (Moser et al. 1989, Gunter and Adlard 2008, 2009, Heiniger et al. 2008, Gunter et al. 2009). During a parasitological survey of fishes collected off Lizard Island, species of Ceratomyxa were found in the gall bladders of Bothus pantherinus (Rüppell), Chaetodon vagabundus Linnaeus, Hyporhamphus dussumieri (Valenciennes), Sphyraena forsteri Cuvier, and Zebrasoma veliferum (Bloch). All of these species are new to science and four species are described herein.

\section{MATERIALS AND METHODS}

Host and parasite collection. Fishes were collected by line, spear-fishing and seine-netting off Lizard Island $\left(14^{\circ} 40^{\prime} \mathrm{S}\right.$, $\left.145^{\circ} 27^{\prime} \mathrm{E}\right)$ in the northern Great Barrier Reef. Fish were euthanised using neural pithing. The gall bladders were removed from the abdominal mass, placed in a cavity block and ruptured. A drop of bile was placed on a glass microscope slide and covered with a glass cover-slip. The slide was examined using a light microscope at $\times 400$ magnification. Infected samples were preserved in $100 \%$ ethanol for DNA analysis and frozen in saline for the measurement of spores.
Morphological analysis of spores. Morphological measurements of spores followed the guidelines devised by Lom and Arthur (1989) for species descriptions of Myxosporea. However, for characterisation of ceratomyxid spores, an additional measurement of the posterior spore angle was made as per Heiniger et al. (2008) and apical width of spores was recorded when possible. If plasmodia were observed, digital images of fresh material were taken in the field. Spores were characterised from frozen material to prevent shrinkage often caused by the use of chemical fixatives. Thirty spores were measured from digital images taken at $\times 400$ magnification using a Nikon Digital Sight DS-L1 (Nikon Corporation, Japan) except for Ceratomyxa koieae sp. n. where only 20 mature spores were characterised due to the small number of mature spores in the sample. Apical width measurements were recorded from as many spores as possible $(\mathrm{n}=0-9)$. Measurements were calibrated using a micrometer. The measurements are given in micrometres $(\mu \mathrm{m})$ as the mean with standard deviation, followed by the range and number of measurements (n) in parentheses.

Small Subunit rDNA analysis. DNA of Ceratomyxa spp. was extracted from $250 \mu \mathrm{l}$ of infected bile preserved in ethanol. The sample was pelleted at $4,000 \mathrm{~g}$ for $10 \mathrm{~min}$ and the ethanol removed. DNA was extracted from the pellet as per the recommended protocol accompanying the QIAgen DNeasy Kit (QIAGEN Inc., Valencia, California). Partial small subunit ribosomal DNA (SSU rDNA) was amplified by PCR using the primers MyxospecF 5, TTC TGC CCT ATC AAC TWG TTG (Fiala 2006) and 18R 5' CTA CGG AAA CCT TGT TAC G (Whipps 
et al. 2003). PCR and sequencing reactions were carried out as described by Heiniger et al. (2008).

Phylogenetic analysis. The SSU rDNA region from the taxa sequenced in this study were edited using BioEdit version 7.0.0 (Hall 1999). Selected SSU rDNA sequences (Table 1) were downloaded from GenBank and included one representative of each Ceratomyxa species available in GenBank. Ellipsomyxa gobii was chosen as the outgroup. An alignment was produced using CLUSTAL W (Thompson et al. 1994), edited by eye and, when trimmed using BioEdit version 7.0.0 (Hall 1999), a 1,664 base alignment was produced. Maximum parsimony (MP) and Neighbour Joining (NJ) analyses were conducted in PAUP* 4.0b 10 (Swofford 2003). MP employed a heuristic search with 10 repetitions of random sequence addition, tree bisection and reconnection branch swapping. NJ analysis was conducted using default parameters of PAUP* 4.0b 10. The strength of the resultant relationships was tested by bootstrap analyses with 1,000 replicates for both MP and NJ analyses. For maximum likelihood (ML), the optimal evolutionary model of nucleotide substitution using Akaike Information Criterion was determined using Modeltest 3.06 (Posada and Crandall 1998) which identified the general time reversible $(\mathrm{GTR}+\mathrm{I}+\mathrm{G})$ model with proportion of invariable sites $=0.271$ and gamma distribution $=0.639$ estimated from the data. ML analysis was performed using PAUP* 4.0b 10 with above parameters. Bootstrap confidence values were calculated with 100 replicates. Bayesian analyses (BA) were conducted in MrBayes (Ronquist and Huelsenbeck 2003) using the evolutionary model as above, with 2 million generations and tree sampling every 100 generations. Graphical and statistical analyses implemented in Tracer 1.4 (http:// beast.bio.ed.ac.uk/Tracer) were used to check the performance and accuracy of MrBayes analysis. Based on the results, the last 10,000 trees were used to build the consensus tree.

\section{RESULTS}

\section{Species descriptions}

\section{Ceratomyxa bartholomewae sp. $\mathrm{n}$.}

Figs. 1A-D, 2A-C, Table 2

Spores (Figs. 1A-D, 2A-C): Mature spore slightly crescent-shaped in lateral view, $5.5 \pm 0.3(4.7-6.2)$ in length and $17.5 \pm 1.8(13.5-21.7)$ in thickness $(\mathrm{n}=30)$. Cylindrical in apical view, $5.4 \pm 0.3(5.0-5.9)$ in width $(\mathrm{n}=8)$. Posterior angle slightly concave to slightly convex $\left(154.6-183.1^{\circ}\right)$. Valves unequal in size, smoothly ovoid in lateral view. Straight suture visible between valves. Spherical polar capsules, $2.3 \pm 0.3(1.8-2.8)$ in length and $2.3 \pm 0.2(1.6-2.7)$ in width $(\mathrm{n}=30)$, positioned centrally in the apex of spore but occasionally off centre in lateral view, centrally located in apical view. Occasional spores with 3 valves observed (See Fig. 1C).

Plasmodia: Disporous (Fig. 1D).

Type host: Hyporhamphus dussumieri (Valenciennes) (Dussumier's halfbeak), Family Hemirhamphidae.

Type locality: Off Lizard Island, Great Barrier Reef, Queensland (14³9'S, $\left.145^{\circ} 27^{\prime} \mathrm{E}\right)$.

Site of infection: Gall bladder.

Prevalence: 1 of $10(10 \%)$.
Type material: Syntypes - air-dried slides stained with Giemsa, registration numbers G465404-465406, deposited in the collections of the Queensland Museum, Brisbane, Australia.

E ty m o log y: In honour of Jerri Bartholomew, Oregon State University, for her research of Ceratomyxa shasta.

Remarks: Ceratomyxa bartholomewae is morphologically similar to Ceratomyxa gibba Meglitsch, 1960, C. priacanthi Kalavati, Dorothy et Pandian, 2002, C. sprenti Moser, Kent et Dennis, 1989 and C. robertsthomsoni Gunter, Whipps et Adlard, 2009. It can be distinguished from $C$. gibba and $C$. priacanthi on the basis of the shape and size of the polar capsules. Ceratomyxa bartholomewae has spherical polar capsules which are smaller $(2.3 \times 2.3 \mu \mathrm{m})$ than $C$. priacanthi $(5.2 \times 2.8 \mu \mathrm{m})$, while the polar capsules of $C$. gibba are pyriform $(2.8 \times 2.3 \mu \mathrm{m})$. Ceratomyxa gibba also has longer spores $(6.9 \mu \mathrm{m})$ than $C$. bartholomewae $(5.5 \mu \mathrm{m})$. The spores of $C$. sprenti are generally thinner, but have a greater length range $(16.3 \times 4-8 \mu \mathrm{m})$ than those of $C$. bartholomewae $(17.5 \times 4.7-6.2 \mu \mathrm{m})$ and the anterior and posterior margins of $C$. sprenti spores are more or less straight while the posterior angle of Ceratomyxa bartholomewae is slightly convex. Ceratomyxa bartholomewae has longer spores $(5.5 \mu \mathrm{m})$ than $C$. robertsthomsoni $(4.7 \mu \mathrm{m})$ but can also be distinguished by SSU rRNA sequence data.

A total of 1,403 bases of SSU rDNA was generated for Ceratomyxa bartholomewae (GenBank accession GU136391). The sequence differs from the aligned sequences of Ceratomyxa spp. at 154-283 of the 1,664 nucleotide alignment and has a maximum genetic similarity of $87.5 \%$ with $C$. dennisi.

This is the first record of a Ceratomyxa infection in a species from the family Hemirhamphidae (Beloniformes).

Ceratomyxa koieae sp. n. Figs. 2D-F, 3A, B, Table 2

Spores (Figs. 2D-F, 3A, B): Mature spore slightly crescent-shaped in lateral view, $5.5 \pm 0.4$ (4.9-6.7) in length and $36.7 \pm 3.4(28.6-41.2)$ in thickness $(n=30)$. Cylindrical in apical view, $5.3 \pm 0.1(5.1-5.4)$ in width $(\mathrm{n}=3)$. Posterior angle slightly concave to slightly convex $\left(156.3-183.8^{\circ}\right)$. Valves almost equal in size, smoothly ovoid in lateral view. Straight suture visible between valves. Subspherical polar capsules, $2.8 \pm 0.2 \quad(2.5-2.9)$ in length and $2.6 \pm 0.2(2.3-2.9)$ in width $(n=30)$, positioned at apex of spore, centrally located in apical view.

Plasmodia: Not observed.

Type host: Sphyraena forsteri Cuvier (bigeye barracuda), Family Sphyraenidae.

Type locality: Off Lizard Island, Great Barrier Reef, Queensland $\left(14^{\circ} 39^{\prime} \mathrm{S}, 145^{\circ} 27^{\prime} \mathrm{E}\right)$.

Site of infection: Gall bladder.

Prevalence: 1 of $2(50 \%)$.

Type material: Syntypes - air-dried slides stained with Giemsa, registration numbers G465407-465409, deposited 

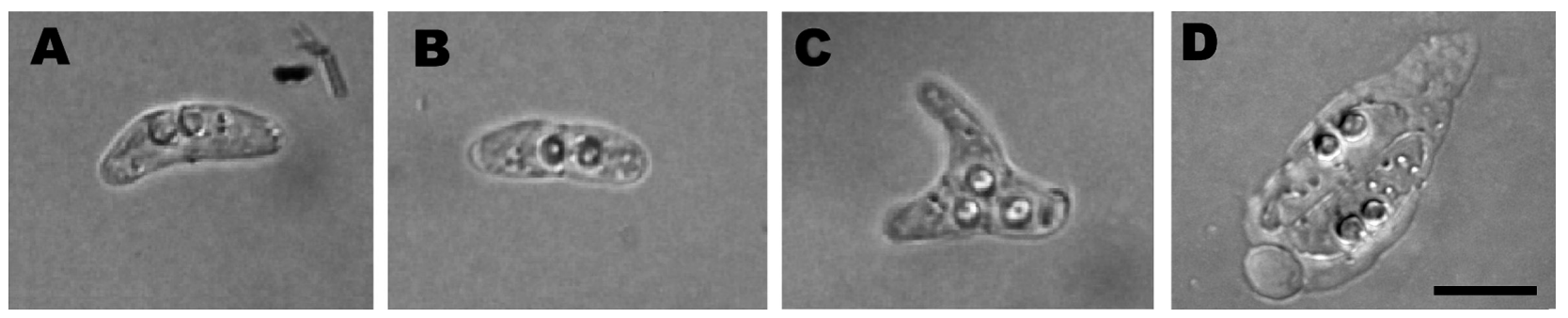

Fig. 1. Ceratomyxa bartholomewae sp. n. from Hyporhamphus dussumieri; photomicrographs of spores. A-sutural view; B - apical view; $\mathbf{C}$ - atypical 3-valved spore; $\mathbf{D}$ - disporous plasmodium. Scale bar $=10 \mu \mathrm{m}$.

A

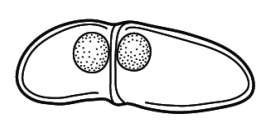

B

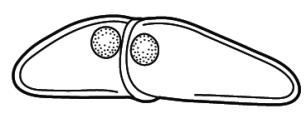

C

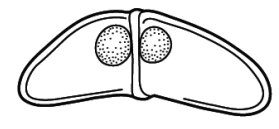

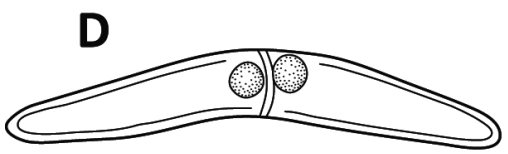

G

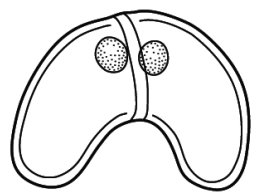

J

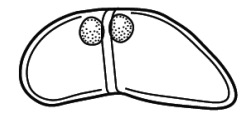

$\mathbf{E}$
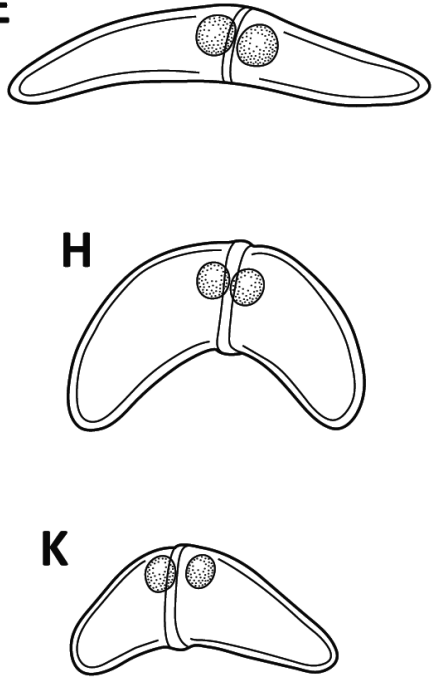

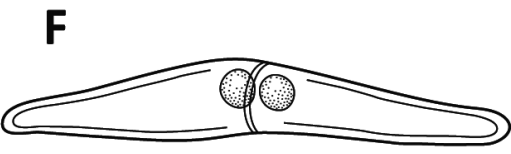

I
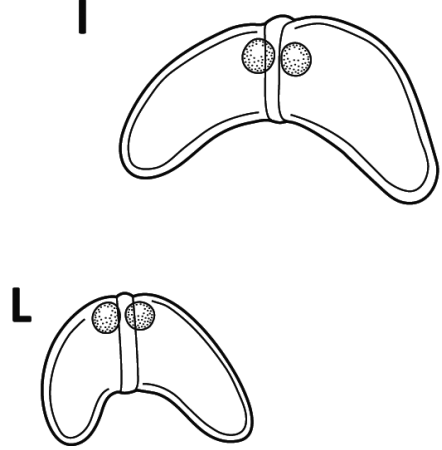

M

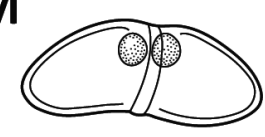

$\mathbf{N}$

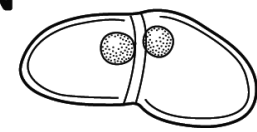

$10 \mu \mathrm{m}$

Fig. 2. Diagrammatic illustrations of morphological variability of spores of new Ceratomyxa species. A-C - Ceratomyxa bartholomewae sp. n.; D-F - Ceratomyxa koieae sp. n.; G-I - Ceratomyxa pantherini $\mathrm{sp.}$.; $\mathbf{J}-\mathbf{L}-$ Ceratomyxa reidi $\mathrm{sp} . \mathrm{n}$.; $\mathbf{M}, \mathbf{N}-$ Ceratomyxa sp. ex Zebrasoma veliferum.
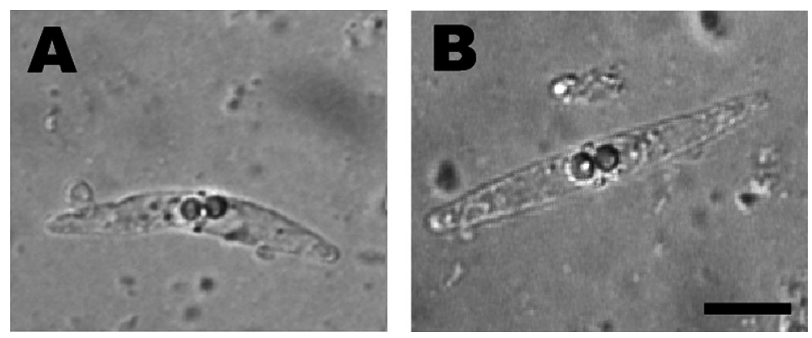

Fig. 3. Ceratomyxa koieae sp. n. from Sphyraena forsteri; photomicrographs of spores. A - sutural view; B - apical view. Scale bar $=10 \mu \mathrm{m}$. 
Table 1. Sequences included in molecular analyses.

\begin{tabular}{|c|c|c|c|}
\hline Species & $\begin{array}{l}\text { GenBank } \\
\text { Acc. No. }\end{array}$ & Host & Reference \\
\hline Ceratomyxa anko & DQ301510 & Lophius litulon (Lophiiformes: Lophiidae) & Freeman et al. 2008 \\
\hline Ceratomyxa atkinsoni & FJ204244 & Lethrinus atkinsoni (Perciformes: Lethrinidae) & Gunter et al. 2009 \\
\hline Ceratomyxa auerbachi & EU616732 & Clupea harengus (Clupeiformes: Clupeidae) & Køie et al. 2008 \\
\hline Ceratomyxa barnesi & FJ204245 & Siganus lineatus (Perciformes: Siganidae) & Gunter et al. 2009 \\
\hline Ceratomyxa bartholomewae sp. $\mathrm{n}$. & GU136391 & Hyporhamphus dussumieri (Beloniformes: Hemirhamphidae) & This paper \\
\hline Ceratomyxa brayi 1 & EU729697 & Cephalopholis boenak (Perciformes: Serranidae) & Gunter and Adlard 2009 \\
\hline Ceratomyxa brayi 2 & EU729699 & Cephalopholis boenak (Perciformes: Serranidae) & Gunter and Adlard 2009 \\
\hline Ceratomyxa bryanti & EU440357 & Abudefduf whitleyi (Perciformes: Pomacentridae) & Gunter and Adlard 2008 \\
\hline Ceratomyxa burgerae & EU440366 & Dascyllus aruanus (Perciformes: Pomacentridae) & Gunter and Adlard 2008 \\
\hline Ceratomyxa capricornensis & EU440364 & Dischistodus perspicillatus (Perciformes: Pomacentridae) & Gunter and Adlard 2008 \\
\hline Ceratomyxa cheilinae & EU045331 & Cheilinus chlorourus (Perciformes: Labridae) & Heiniger et al. 2008 \\
\hline Ceratomyxa choerodonae & EU045333 & Choerodon cyanodus (Perciformes: Labridae) & Heiniger et al. 2008 \\
\hline Ceratomyxa cribbi & EU440367 & Pomacentrus chrysurus (Perciformes: Pomacentridae) & Gunter and Adlard 2008 \\
\hline Ceratomyxa cutmorei & EU729691 & Epinephelus fasciatus (Perciformes: Serranidae) & Gunter and Adlard 2009 \\
\hline Ceratomyxa diamanti & FJ204246 & Polydactylus macrochir (Perciformes: Polydactylidae) & Gunter et al. 2009 \\
\hline Ceratomyxa dennisi & EU440358 & Acanthochromis polyacanthus (Perciformes: Pomacentridae) & Gunter and Adlard 2008 \\
\hline Ceratomyxa ernsti & FJ204247 & Sillago ciliata (Perciformes: Sillaginidae) & Gunter et al. 2009 \\
\hline Ceratomyxa falcatus & EU440361 & Abudefduf whitleyi (Perciformes: Pomacentridae) & Gunter and Adlard 2008 \\
\hline Ceratomyxa gleesoni & EU729693 & Plectropomus leopardus (Perciformes: Serranidae) & Gunter and Adlard 2009 \\
\hline Ceratomyxa hallettae & FJ204248 & Lethrinus harak (Perciformes: Lethrinidae) & Gunter et al. 2009 \\
\hline Ceratomyxa heinigerae & FJ204249 & Choerodon cephalotes (Perciformes: Labridae) & Gunter et al. 2009 \\
\hline Ceratomyxa hooperi & EU729692 & Epinephelus quoyanus (Perciformes: Serranidae) & Gunter and Adlard 2009 \\
\hline Ceratomyxa jonesi & FJ204250 & Pseudolabrus guentheri (Perciformes: Labridae) & Gunter et al. 2009 \\
\hline Ceratomyxa kenti & EU440363 & Abudefduf sexfasciatus (Perciformes: Pomacentridae) & Gunter and Adlard 2008 \\
\hline Ceratomyxa koieae sp. $\mathrm{n}$. & GU136392 & Sphyraena forsteri (Perciformes: Sphyraenidae) & This paper \\
\hline Ceratomyxa labracis & AF411472 & Dicentrarchus labrax (Perciformes: Moronidae) & Palenzuela et al. 2002 \\
\hline Ceratomyxa lunula 1 & EU440376 & Neoglyphidon melas (Perciformes: Pomacentridae) & Gunter and Adlard 2008 \\
\hline Ceratomyxa lunula 2 & EU440377 & Neoglyphidon melas (Perciformes: Pomacentridae) & Gunter and Adlard 2008 \\
\hline Ceratomyxa lunula 3 & EU440378 & Neoglyphidon melas (Perciformes: Pomacentridae) & Gunter and Adlard 2008 \\
\hline Ceratomyxa milleri & FJ204251 & Lutjanus fulviflamma (Perciformes: Lutjanidae) & Gunter et al. 2009 \\
\hline Ceratomyxa moseri & EU440360 & Pomacentrus wardi (Perciformes: Pomacentridae) & Gunter and Adlard 2008 \\
\hline Ceratomyxa nolani 1 & EU729695 & Epinephelus quoyanus (Perciformes: Serranidae) & Gunter and Adlard 2009 \\
\hline Ceratomyxa nolani 2 & EU729698 & Epinephelus quoyanus (Perciformes: Serranidae) & Gunter and Adlard 2009 \\
\hline Ceratomyxa nowakae & FJ204252 & Gymnocranius audleyi (Perciformes: Lethrinidae) & Gunter et al. 2009 \\
\hline Ceratomyxa oxycheilinae & EU045334 & Oxycheilinus digramma (Perciformes: Labridae) & Heiniger et al. 2008 \\
\hline Ceratomyxa pantherini $\mathrm{sp} . \mathrm{n}$. & GU136393 & Bothus pantherinus (Pleuronectiformes: Bothidae) & This paper \\
\hline Ceratomyxa reidi $\mathrm{sp} . \mathrm{n}$. & GU136394 & Chaetodon vagabundus (Perciformes: Chaetodontidae) & This paper \\
\hline Ceratomyxa robertsthomsoni & FJ204253 & Liza vaigiensis (Mugiliformes: Mugilidae) & Gunter et al. 2009 \\
\hline Ceratomyxa sewelli & EU440362 & Pomacentrus wardi (Perciformes: Pomacentridae) & Gunter and Adlard 2008 \\
\hline Ceratomyxa shasta & AF001579 & Oncorhynchus mykiss (Salmoniformes: Salmonidae) & Bartholomew et al. 1997 \\
\hline Ceratomyxa sp. & GU136395 & Zebrasoma veliferum (Perciformes: Acanthuridae) & This paper \\
\hline Ceratomyxa sp. M0304 & FJ417043 & Gadus morhua (Gadiformes: Gadidae) & Bartošová et al. 2009 \\
\hline Ceratomyxa sp. RM & DQ333429 & Siganus rivulatus (Perciformes: Siganidae) & - \\
\hline Ceratomyxa sp. SAR & DQ333430 & Siganus rivulatus (Perciformes: Siganidae) & - \\
\hline Ceratomyxa sp. SRR & DQ333431 & Siganus rivulatus (Perciformes: Siganidae) & - \\
\hline Ceratomyxa sp. VL & DQ333432 & Variola louti (Perciformes: Serranidae) & - \\
\hline Ceratomyxa sp. 1 & DQ377698 & Scyliorhinus canicula (Carcharhiniformes: Scyliorhinidae) & Fiala 2006 \\
\hline Ceratomyxa sp. 2 & DQ377699 & Notacanthus bonaparte (Notacanthiformes: Notacanthidae) & Fiala 2006 \\
\hline Ceratomyxa sparusaurati & AF411471 & Sparus aurata (Perciformes: Sparidae) & Palenzuela et al. 2002 \\
\hline Ceratomyxa talboti & EU440368 & Dischistodus chrysopoecilus (Perciformes: Pomacentridae) & Gunter and Adlard 2008 \\
\hline Ceratomyxa thalassomae & EU045332 & Thalassoma lunare (Perciformes: Labridae) & Heiniger et al. 2008 \\
\hline Ceratomyxa vikrami* & FJ417044 & Zeus faber (Zeiformes: Zeidae) & Bartošová et al. 2009 \\
\hline Ceratomyxa whippsi & EU729694 & Cephalopholis boenak (Perciformes: Serranidae) & Gunter and Adlard 2009 \\
\hline Ceratomyxa yokoyamai & EU729696 & Epinephelus maculatus (Perciformes: Serranidae) & Gunter and Adlard 2009 \\
\hline Ellipsomyxa gobii & AY505126 & Pomatoschistus microps (Perciformes: Gobiidae) & Køie et al. 2004 \\
\hline Palliatus indecorus & DQ377712 & Alepocephalus grandis (Osmeriformes: Alepocephalidae) & Fiala 2006 \\
\hline
\end{tabular}

* Transferred from Leptotheca to Ceratomyxa by Gunter and Adlard (2010). 
Table 2. Morphometrics of new Ceratomyxa species. Mean spore dimensions in $\mu \mathrm{m}$ or ${ }^{\circ}$ (range in parentheses). $\mathrm{L}-$ length; $\mathrm{T}-$ thickness; S - sutural position; S\% - percentage thickness of smaller valve (S); W - width; PCL - polar capsule length; PCW - polar capsule width; PA - posterior angle (dimension is in ${ }^{\circ}$ ).

\begin{tabular}{|c|c|c|c|c|c|c|c|c|c|}
\hline Ceratomyxa species & Host species & $\mathrm{L}$ & $\mathrm{T}$ & $\mathrm{S}$ & $\mathrm{S} \%$ & W & PCL & PCW & PA \\
\hline $\begin{array}{l}\text { Ceratomyxa } \\
\text { bartholomewae sp. n. }\end{array}$ & $\begin{array}{l}\text { Hyporhamphus } \\
\text { dussumieri }\end{array}$ & $\begin{array}{l}5.5 \\
(4.7-6.2)\end{array}$ & $\begin{array}{l}17.5 \\
(13.5-21.7)\end{array}$ & $\begin{array}{l}7.6 \\
(5.6-9.9)\end{array}$ & $\begin{array}{l}43.4 \\
(36.9-50)\end{array}$ & $\begin{array}{l}5.4 \\
(5.0-5.9)\end{array}$ & $\begin{array}{l}2.3 \\
(1.8-2.8)\end{array}$ & $\begin{array}{l}2.3 \\
(1.6-2.7)\end{array}$ & $\begin{array}{l}168.5 \\
(154.6-183.1)\end{array}$ \\
\hline $\begin{array}{l}\text { Ceratomyxa } \\
\text { koieae sp. } \mathrm{n} .\end{array}$ & $\begin{array}{l}\text { Sphyraena } \\
\text { forsteri }\end{array}$ & $\begin{array}{l}5.5 \\
(4.9-6.7)\end{array}$ & $\begin{array}{l}36.7 \\
(28.5-41.2)\end{array}$ & $\begin{array}{l}17.5 \\
(13.8-20.0)\end{array}$ & $\begin{array}{l}47.8 \\
(43.7-49.9)\end{array}$ & $\begin{array}{l}5.3 \\
(5.1-5.4)\end{array}$ & $\begin{array}{l}2.8 \\
(2.5-3.2)\end{array}$ & $\begin{array}{l}2.6 \\
(2.3-2.9)\end{array}$ & $\begin{array}{l}168.7 \\
(156.3-183.8)\end{array}$ \\
\hline $\begin{array}{l}\text { Ceratomyxa } \\
\text { pantherini sp. } \mathrm{n} .\end{array}$ & $\begin{array}{l}\text { Bothus } \\
\text { pantherinus }\end{array}$ & $\begin{array}{l}8.1 \\
(7.3-9.1)\end{array}$ & $\begin{array}{l}21.6 \\
(17.9-24.6)\end{array}$ & $\begin{array}{l}10.1 \\
(8.4-11.9)\end{array}$ & $\begin{array}{l}46.8 \\
(41.5-49.8)\end{array}$ & $\begin{array}{l}8.0 \\
(7.1-8.9)\end{array}$ & $\begin{array}{l}2.3 \\
(1.8-2.6)\end{array}$ & $\begin{array}{l}2.2 \\
(1.7-2.6)\end{array}$ & $\begin{array}{l}119.1 \\
(85.7-156.7)\end{array}$ \\
\hline $\begin{array}{l}\text { Ceratomyxa } \\
\text { reidi } \mathrm{sp} . \mathrm{n} .\end{array}$ & $\begin{array}{l}\text { Chaetodon } \\
\text { vagabundus }\end{array}$ & $\begin{array}{l}6.8 \\
(5.8-7.5)\end{array}$ & $\begin{array}{l}17.5 \\
(14.3-20.7)\end{array}$ & $\begin{array}{l}7.9 \\
(6.5-9.2)\end{array}$ & $\begin{array}{l}45.1 \\
(39.8-48.6)\end{array}$ & $\begin{array}{l}6.5 \\
(6.0-7.0)\end{array}$ & $\begin{array}{l}2.1 \\
(1.7-2.4)\end{array}$ & $\begin{array}{l}2.0 \\
(1.7-2.5)\end{array}$ & $\begin{array}{l}141.2 \\
(102.9-170.3)\end{array}$ \\
\hline
\end{tabular}

Table 3. Morphometric comparison of Ceratomyxa species from bothid fishes. Bold measurements do not overlap with those of C. pantherini. Bold italicised measurement ranges overlap partially with those of $C$. pantherini but means are distinct. Normal text measurements are superficially similar to $C$. pantherini. For abbreviations see Table 2 .

\begin{tabular}{|c|c|c|c|c|c|c|}
\hline Ceratomyxa species & $\mathrm{L}$ & $\mathrm{T}$ & W & PCL & PCW & PAA \\
\hline C. pantherini sp. n. & $8.1(7.3-9.1)$ & $21.60(17.9-24.6)$ & $8.0(7.1-8.9)$ & $2.3(1.8-2.6)$ & $2.3(1.7-2.6)$ & $119(86-157)$ \\
\hline C. faba Meglitsch, 1960 & $6.2(5.6-6.7)$ & $12.7(10.7-14.1)$ & $6.4(6.1-7.0)$ & $2.4(2.0-3.1)$ & $2.4(2.0-3.1)$ & $162(125-180)$ \\
\hline C. hama Meglitsch, 1960 & $7.4(6.8-8.3)$ & $23.4(18.5-29.8)$ & $7.7(7.0-8.3)$ & $3.2(2.0-3.5)$ & $3.2(2.0-3.5)$ & $111(89-152)$ \\
\hline C. laxa Meglitsch, 1960 & $8.7(7.8-9.8)$ & $25.6(20.8-30.3)$ & - & $3.4(2.9-3.9)$ & $3.4(2.9-3.9)$ & $130(93-173)$ \\
\hline C. renalis* Meglitsch, 1960 & $8.0(7.3-8.5)$ & $27.7(22.5-33.4)$ & $8.9(7.7-9.3)$ & $3.1(2.7-3.2)$ & $3.1(2.7-3.2)$ & $118(90-134)$ \\
\hline C. torquata Meglitsch, 1960 & $8.1(7.8-9.5)$ & $26.9(20.5-30.3)$ & $9.2(8.3-10.1)$ & $3.8(2.8-4.7)$ & $3.8(2.8-4.7)$ & $135(115-178)$ \\
\hline C. vepallida Meglitsch, 1960 & $8.6(7.8-9.6)$ & $18.7(16.0-21.4)$ & 8.1 & $3.2(2.9-3.6)$ & $2.9(2.5-3.2)$ & $158(142-180)$ \\
\hline
\end{tabular}

* C. renalis infects the urinary bladder.

in the collections of the Queensland Museum, Brisbane, Australia.

E t y m o l o g y: In honour of Marianne Køie, University of Copenhagen, Denmark, for her research into marine myxosporean life cycles.

Remarks: Ceratomyxa koieae is superficially similar to Ceratomyxa epinepheli Wu, Wu et Dingke, 1993, C. huanghaiensis Zhao et Song, 2003 and C. urophysis Fantham, Porter et Richardson, 1940. Ceratomyxa epinepheli has shorter spores with smaller polar capsules $(5.3 ; 2.6 \times 2.6 \mu \mathrm{m})$ than $C$. koieae $(5.5 ; 2.8 \times 2.8 \mu \mathrm{m})$. Ceratomyxa koieae has shorter spores $(5.5 \mu \mathrm{m})$ than C. huanghaiensis $(6.5 \mu \mathrm{m})$. Also, the valves of $C$. huanghaiensis are unequal. The description of C. urophysis only contains ranges for spore length and thickness (5-7.5 $\times 25-39.1 \mu \mathrm{m})$ and both these measurements overlap with those of $C$. koieae $(4.9-6.7 \times 28.5-41 \mu \mathrm{m})$ but the figure depicts $C$. urophysis with pyriform polar capsules which are distinct from the subspherical polar capsules of $C$. koieae.

A total of 1,379 bases of SSU rDNA was generated for Ceratomyxa koieae (GenBank accession GU136392). The sequence differs from the aligned sequences of Ceratomyxa spp. at 148-299 of the 1,664 nucleotide alignment and has a maximum genetic similarity of $87.7 \%$ with $C$. jonesi. Ceratomyxa koieae is the first described species of this genus recorded from a species of Sphyraenidae (Perciformes).

\section{Ceratomyxa pantherini $\mathrm{sp} . \mathrm{n}$.}

Figs. 2G-I, 4A-D, Table 2

Spores (Figs. 2G-I, 4A-D): Mature spore slightly crescent-shaped in lateral view, $8.1 \pm 0.4$ (7.3-9.1) in length and $21.6 \pm 2.0 \quad(17.9-24.6)$ in thickness $(\mathrm{n}=30)$. Cylindrical or rarely sigmoid in apical view, $8.0 \pm 0.5$ (7.1-8.9) in width $(n=9)$. Posterior angle strongly to moderately concave $\left(85.7-156.7^{\circ}\right)$. Valves almost equal in size, smoothly rounded in lateral view. Straight suture visible between valves. Subspherical polar capsules, $2.3 \pm$ $0.2(1.8-2.6)$ in length and $2.2 \pm 0.2(1.7-2.6)$ in width $(n=30)$, positioned at apex of spore. In apical view the polar capsules are not located centrally but are positioned touching one wall of the valves (see Fig. 4B).

Plasmodia: Disporous and polysporous (Fig. 4 C, D).

Type host: Bothus pantherinus (Rüppell) (leopard flounder), Family Bothidae.

Type locality: Off Lizard Island, Great Barrier Reef, Queensland $\left(14^{\circ} 39^{\prime} \mathrm{S}, 145^{\circ} 27^{\prime} \mathrm{E}\right)$.

Site of infection: Gall bladder.

Prevalence: 1 of $1(100 \%)$.

Type material: Syntypes - air-dried slides stained with Giemsa, registration numbers G465410-465412, deposited in the collections of the Queensland Museum, Brisbane, Australia.

E ty m ology: Specific name refers to the host species. 

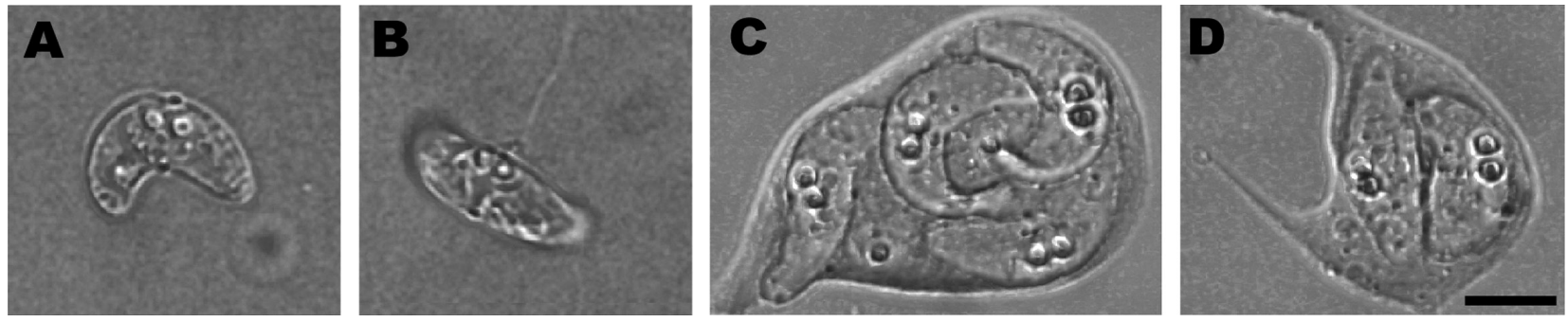

Fig. 4. Ceratomyxa pantherini sp. n. from Bothus pantherinus; photomicrographs of spores. A - sutural view; B - apical view, note polar capsules are not central; $\mathbf{C}$ - polysporous plasmodium; $\mathbf{D}$-disporous plasmodium. Scale bar $=10 \mu \mathrm{m}$.

Remarks: Ceratomyxa pantherini is the first species described in the genus with polar capsules not located centrally in apical view. It is superficially similar to Ceratomyxa artedielli Polyanskii, 1955, C. blennius Noble, 1938, C. diloba Dogiel, 1948, C. hama Meglitsch, 1960, C. lagocephali Kpatcha, Diebakate, Faye et Toguebaye, 1996 and C. laxa Meglitsch, 1960. It can be distinguished from all these species on the basis of the shape and size of the polar capsules. The polar capsules of C. pantherini are smaller $(2.3 \times 2.2 \mu \mathrm{m})$ than those of $C$. artedielli $(3.6 \times 3.6 \mu \mathrm{m})$, C. blennius $(3 \times 3 \mu \mathrm{m})$, C. diloba $(3 \times 3 \mu \mathrm{m})$, C. hama $(3.2 \times 3.5 \mu \mathrm{m})$, C. lagocephali $(4.2 \times 4.5 \mu \mathrm{m})$ and $C$. $\operatorname{laxa}(3.4 \times 3.4 \mu \mathrm{m})$.

A total of 1,489 bases of SSU rDNA was generated for Ceratomyxa pantherini (GenBank accession GU136393). The sequence differs from the aligned sequences of $\mathrm{Cer}$ atomyxa spp. at 206-367 of the 1,664 nucleotide alignment and has a maximum genetic similarity of $83.6 \%$ with C. anko.

Only one other species of bothid is known to harbour a species of Ceratomyxa. Arnoglossus scapha (= Caulopsetta scapha) is host to seven species: C. faba, C. hama, C. laxa, C. renalis, C. torquata, C. uncinata, and C. vellapida (see Meglitsch 1960), the richest fauna of Ceratomyxa known from a single host. Although all species overlap slightly, each can be distinguished from C. pantherini (Table 3).

Ceratomyxa reidi sp. n. Figs. 2J-L, 5A, B, Table 2

Spores (Figs. 2J-L, 5A, B): Mature spore slightly crescent-shaped in lateral view, $6.8 \pm 0.4(5.8-7.5)$ in length and $17.5 \pm 1.5(14.3-20.7)$ in thickness $(\mathrm{n}=30)$. Cylindrical in apical view, $6.5 \pm 0.4(6.0-7.0)$ in width $(\mathrm{n}=7)$. Posterior angle strongly to slightly concave (102.9$170.3^{\circ}$ ). Valves slightly unequal in size, smoothly ovoid in lateral view. Straight suture visible between valves. Subspherical polar capsules, $2.1 \pm 0.2(1.7-2.4)$ in length and $2.0 \pm 0.2(1.7-2.5)$ in width $(\mathrm{n}=30)$, positioned at apex of spore, centrally located in apical view.

Plasmodia: Monosporous.

Ty p e host: Chaetodon vagabundus Linnaeus (Indian vagabond butterflyfish), Family Chaetodontidae.
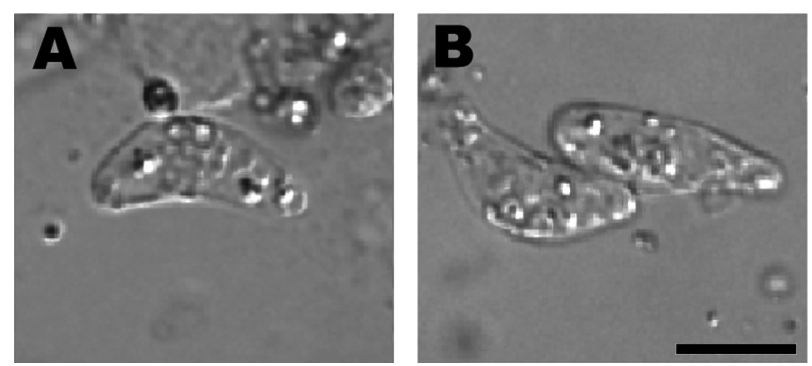

Fig. 5. Ceratomyxa reidi sp. n. from Chaetodon vagabundus; photomicrographs of spores. A - sutural view; B - apical view. Scale bar $=10 \mu \mathrm{m}$.

Type locality: Off Lizard Island, Great Barrier Reef, Queensland $\left(14^{\circ} 39^{\prime} \mathrm{S}, 145^{\circ} 27^{\prime} \mathrm{E}\right)$.

Site of infection: Gall bladder.

Prevalence: 5 of $22(22.7 \%)$.

Type material: Syntypes - air-dried slides stained with Giemsa, registration numbers G465413-465415, deposited in the collections of the Queensland Museum, Brisbane, Australia.

E t y m o log y: In honour of Anthony Reid, The University of Queensland.

Remarks: Ceratomyxa reidi is superficially similar to Ceratomyxa cottoidi Reed, Basson, Van As et Dyková, 2007, C. cutmorei Gunter et Adlard, 2009, C. gibba and C. lateolabracis Zhao et Song, 2003. Ceratomyxa reidi has thicker spores and smaller polar capsules (17.5; $2.1 \times 2.0 \mu \mathrm{m})$ than $C$. cutmorei $(16.1 ; 2.4 \times 2.3 \mu \mathrm{m})$ and $C$. gibba $(17 ; 2.8 \times 2.3 \mu \mathrm{m})$. Ceratomyxa cottoidi can be distinguished by having thicker spores and larger polar capsules $(18.2 ; 2.7 \times 2.4 \mu \mathrm{m})$ than $C$. reidi $(17.5$; $2.1 \times 2.0 \mu \mathrm{m})$. The spores of $C$. lateolabracis are thinner $(16.7 \mu \mathrm{m})$ than $C$. reidi $(17.5 \mu \mathrm{m})$ and can also be distinguished by being more ovoid compared to the spores of C. reidi, which taper to a rounded point.

A total of 1,493 bases of SSU rDNA was generated for Ceratomyxa reidi (GenBank accession GU136394) and was obtained from a single infection only. The sequence differs from the aligned sequences of Ceratomyxa spp. at 145-346 of the 1,664 nucleotide alignment and has a maximum genetic similarity of $88.7 \%$ with the un- 

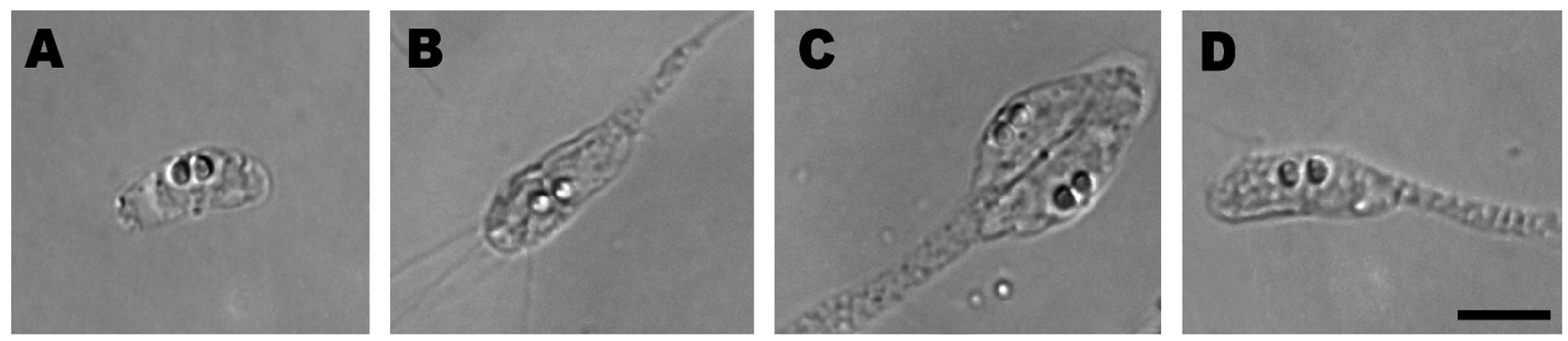

Fig. 6. Ceratomyxa sp. from Zebrasoma veliferum; photomicrographs of spores. A - sutural view; B - immature spore apical view; $\mathbf{C}-$ disporous plasmodium; $\mathbf{D}-$ monosporous plasmodium. Scale bar $=10 \mu \mathrm{m}$.

described species of Ceratomyxa ex Zebrasoma veliferum reported in this study.

Only one other Ceratomyxa species has been described from chaetodontids. Ceratomyxa sprenti Moser, Kent et Dennis, 1989 was described from Chaetodon aureofasciatus and C. rainfordi, but also from fishes of other families: Lutjanus amabilis (Lutjanidae), Platycephalus bosschei (Platycephalidae) and Choerodon venustus (Labridae) off Heron Island, Australia. Although, both species are found in chaetodontids collected from the Great Barrier Reef they are morphologically distinct. On average, the spores of $C$. reidi are longer, thicker and wider $(6.8 \times 17.5 \times 7.9 \mu \mathrm{m})$ than $C$. sprenti $(5.7 \times 16.3 \times$ $3.5-5 \mu \mathrm{m})$ and the polar capsules of $C$. reidi are slightly smaller $(2.1 \times 2.0 \mu \mathrm{m})$ than those of $C$. sprenti $(2.4$ $\times 2.4 \mu \mathrm{m})$. On the basis of morphometric and host data one can conclude that these species are distinct.

\section{Ceratomyxa sp. ex Zebrasoma veliferum}

Spores and plasmodia of Ceratomyxa sp. were found in the gall bladder of 1 of $1(100 \%)$ individual of Zebrasoma veliferum (Bloch) (sailfin tang), Family Acanthuridae, collected off Lizard Island, Great Barrier Reef, Queensland $\left(14^{\circ} 39^{\prime} \mathrm{S}, 145^{\circ} 27^{\prime} \mathrm{E}\right)$. Unfortunately, since infection mainly consisted of immature spores in plasmodia (Fig. 6B-D), insufficient material (mature spores) was available for a thorough morphometric characterisation; thus, we prefer not to describe it as a new species at this time. Only three mature spores free of plasmodia were observed. These spores were elongate ovoid in lateral view, $6.2(5.9-6.8) \mu \mathrm{m}$ in length and $16.8(15.9-17.6) \mu \mathrm{m}$ in thickness $(\mathrm{n}=3)$ (Figs. 2M, N, 6A). Valves slightly unequal in size, smoothly ovoid in lateral view. Straight suture visible between valves. Subspherical polar capsules, $2.4(2.3-2.5) \mu \mathrm{m}$ in length and $2.4(2.3-2.5) \mu \mathrm{m}$ in width $(\mathrm{n}=3)$, positioned at apex of spore, centrally located in apical view (Fig. 6B).

The plasmodia were disporous and monosporous (Fig. 6C, D). A total of 1,426 bases of SSU rDNA was generated for Ceratomyxa sp. ex Zebrasoma veliferum (GenBank accession GU136395). The sequence differs from the aligned sequences of Ceratomyxa spp. at 145-
316 of the 1,664 nucleotide alignment and has a maximum genetic similarity of $88.7 \%$ with Ceratomyxa reidi.

Material: Voucher specimens - air-dried spores on slides stained with Giemsa, registration numbers G465416465417, deposited in the collections of the Queensland Museum, Brisbane, Australia.

Remarks: Only one other species of Ceratomyxa has been described from an acanthurid. Ceratomyxa acanthuri Kpatcha, Diebakate, Faye et Toguebaye, 1996 was described from the gall bladder of Acanthurus monroviae off the Senegal coast. The spores of $C$. acanthuri are superficially similar to the Ceratomyxa sp. ex Zebrasoma veliferum. Although the thickness of the spores are similar $(16.5 \mu \mathrm{m}$ C. acanthuri, 16.8 Ceratomyxa sp. ex Zebrasoma veliferum), the spores of $C$. acanthuri are longer $(10.5 \mu \mathrm{m})$ and the polar capsules are also slightly larger $(2.75 \times 2.75 \mu \mathrm{m})$ than Ceratomyxa sp. ex Zebrasoma veliferum $(6.2 \mu \mathrm{m}$ and $2.4 \times 2.4 \mu \mathrm{m})$. On the basis of host and slight morphometric differences we believe the species from Zebrasoma veliferum is not $C$. acanthuri but represents a distinct and novel species.

\section{Molecular phylogeny}

Sequences from 50 Ceratomyxa species were included in the molecular analyses. Neighbour joining, parsimony, maximum likelihood and Bayesian analyses produced trees with similar topology but had varying levels of clade support (see Fig. 7). The phylogenetic position of Ceratomyxa species and resolution of clades was near identical to that reported in the recent phylogeny of Ceratomyxa (Gunter et al. 2009). Few relationships between species are strongly supported in all analyses because alternate relationships with other species are just as likely.

\section{DISCUSSION}

Since the incorporation of molecular systematics into myxosporean taxonomy, SSU rDNA data have shown that morphologically similar species are not necessarily closely related and other biological characteristics may be important descriptive characters that correlate with genetic 


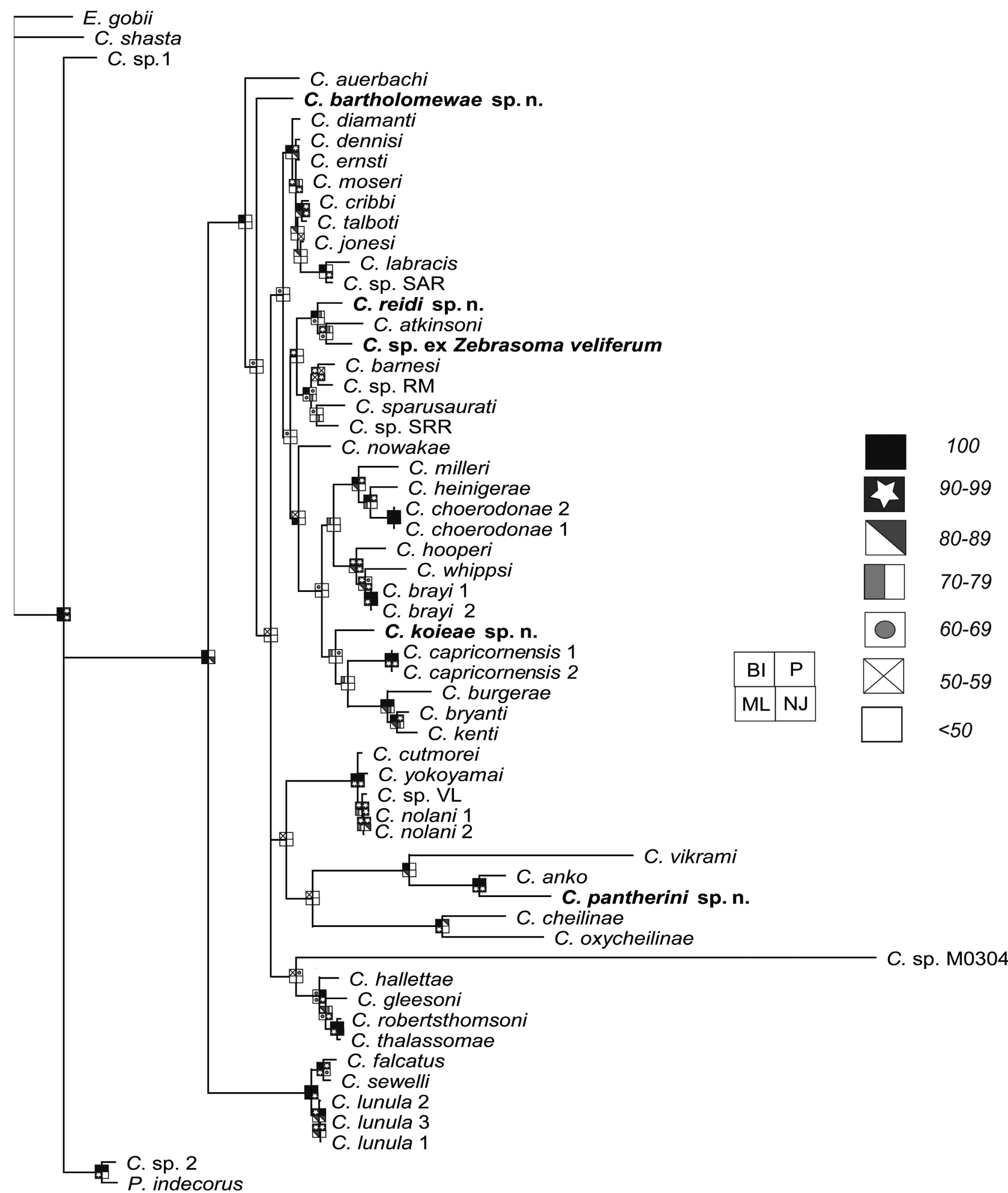

$\underline{0.1}$

Fig. 7. Phylogenetic tree resulting from Bayesian analysis inferred from the SSU rDNA data-set. Support values at branching points are indicated in shades from black $(100)$ to white $(<50)$ and symbols representing intervals of 10 in the nodal boxes as: clade credibility values (CCV) from Bayesian inference (BI: top left); bootstrap values from maximum likelihood (ML: bottom left); bootstrap values from parsimony analyses ( $\mathrm{P}$ : top right) and bootstrap values from neighbour joining analyses (NJ: bottom right). 
relatedness (Whipps et al. 2004, Fiala 2006, Gunter et al. 2009). Tissue tropism is an informative characteristic for discrimination of species in the genera Myxobolus, Henneguya and Kudoa (see Burger et al. 2006, Fiala 2006), but is uninformative for genera that primarily infect a single tissue, i.e. Ceratomyxa and Coccomyxa, species of which are typically restricted to the gall bladder. Molecular studies have revealed that Myxobolus and Ceratomyxa species tend to be highly host specific (Gunter et al. 2009, Molnár et al. 2009) and as such host identity may represent an important diagnostic character. The Ceratomyxa species characterised here are from distantly related families of fishes, each Ceratomyxa species is genetically distinct and appears to be restricted to a single host species. These results support the hypothesis that Ceratomyxa are generally highly host specific. It is becoming more apparent that host species is an informative character for species discrimination in ceratomyxids. Ideally, modern taxonomy should incorporate molecular characterisation, however, not all workers in the field have this capacity. The utility of host as an informative diagnostic character is particularly valuable as species discrimination based on spore morphology alone lacks a level of confidence (particularly in species-rich genera) due to a relative paucity of morphometric characters together with small spore size and morphological plasticity.

The five species characterised here using 18S rRNA form close relationships with other sequenced Ceratomyxa species (see Fig. 7). The position of C. bartholomewae remains unresolved possibly due to it being the only parasite of beloniformes in the analysis. Ceratomyxa reidi and Ceratomyxa sp. ex Zebrasoma veliferum form a supported clade with $C$. atkinsoni, a species from a lethrinid also from Lizard Island. Ceratomyxa koieae sits basally in a clade of pomacentrid-infecting Ceratomyxa spp. While C. pantherini forms a clade with C. anko, C. vikrami, $C$. cheilinae and $C$. oxycheilinae, but only the relationship with $C$. anko is supported in all analyses. Two spe- cies described here share taxonomic affinities with other Ceratomyxa species included in the molecular analyses. Superficially, C. bartholomewae and C. robertsthomsoni are similar, as are $C$. reidi and $C$. cutmorei. However in both cases, neither pair of morphologically similar species is more closely related to each other than to other Ceratomyxa in the analyses.

The molecular analyses contain Ceratomyxa species from a diverse range of fishes including representatives from 12 orders. The vast majority of species are from perciform fishes but the dataset includes a single species from a beloniform, a carcharhiniform, a clupeiform, a gadiform, a lophiiform, a mugilform, a notacanthiform, an osmeriform, a pleuronectiform, a salmoniform and a zeiform. Interestingly, the position of some Ceratomyxa species from non-perciform fishes remains relatively unresolved (e.g. C. auerbachi from a clupeiform, C. bartholomewae from a beloniform, Ceratomyxa sp. M0304 from a gadiform, C. vikrami from a zeiform). Ceratomyxa pantherini and $C$. anko always form a strong relationship and in Bayesian analysis and maximum likelihood also form a clade with C. vikrami; these species infect a pleuronectiform, a lophiiform and a zeiform, respectively. These relationships raise questions about the evolution and speciation of Ceratomyxa. Further research is needed to investigate whether radiation associated with radiation among fish orders is occurring and the inclusion of more species from a diverse range of hosts may also improve the resolution of these clades.

Acknowledgements. We thank Dr. Tom Cribb, Abigail Downie, Ricky Gleeson and Marissa McNamara who assisted in field collection and the staff at Lizard Island Research Station, a facility of the Australian Museum, Sydney. This paper is a contribution from the CReefs Australia Project, generously sponsored by BHP Billiton in partnership with the Australian Biological Resources Study, The Great Barrier Reef Foundation, the Australian Institute of Marine Science and the Alfred P. Sloan Foundation. CReefs is a field program of the Census of Marine Life.

\section{REFERENCES}

Bartholomew J.L., Whipple M.J., Fryer J.L. 1997: The life cycle of Ceratomyxa shasta, a myxosporean parasite of salmonids, requires a freshwater polychaete as an alternate host. J. Parasitol. 83: 859-869.

Bartošová P., Fiala I., Hypša V. 2009: Concatenated SSU and LSU rDNA data confirm the main evolutionary trends within myxosporeans (Myxozoa: Myxosporea) and provide an effective tool for their molecular phylogenetics. Mol. Phylogenet. Evol. 53: 81-93.

Burger M.A.A., Cribb T.H., Adlard R.D. 2007: Patterns of relatedness in the Kudoidae with descriptions of Kudoa chaetodoni sp. n. and K. lethrini sp. n. (Myxosporea: Multivalvulida). Parasitology 134: 669-681.

Fiala I. 2006: The phylogeny of the Myxosporea (Myxozoa) based on small subunit ribosomal RNA gene analysis. Int. J. Parasitol. 36: $1521-1534$.
Freeman M.A., Yokoyama H., Ogawa K. 2008: Description and phylogeny of Ceratomyxa anko sp. $\mathrm{n}$. and Zschokkella lophii sp. n. from the Japanese anglerfish, Lophius litulon (Jordan). J. Fish Dis. 31: 921-930.

Gunter N.L., Adlard R.D. 2008: Bivalvulidan (Myxozoa: Myxosporea) parasites of damselfishes with description of twelve novel species from Australia's Great Barrier Reef. Parasitology 135: 1165-1178.

Gunter N.L., Adlard R.D. 2009: Seven new species of Ceratomyxa from the gall bladders of serranids from the Great Barrier Reef, Australia. Syst. Parasitol. 73: 1-11.

Gunter N., Adlard R. 2010: The demise of the genus Leptotheca Thélohan, 1895 (Myxozoa: Myxosporea: Ceratomyxidae) and assignation of the species to Ceratomyxa Thélohan, 1892 (Myxosporea: Ceratomyxidae), Ellipsomyxa Køie, 2003 (Myxosporea: Ceratomyxidae), Myxobolus Bütschli, 1882 and 
Sphaerospora Thélohan, 1892 (Myxosporea: Sphaerosporidae). Syst. Parasitol. 75: 81-104.

Gunter N.L., Whipps C.M., Adlard R.D. 2009: Ceratomyxa (Myxozoa: Bivalvulida): robust taxon or genus of convenience? Int. J. Parasitol. 39: 1395-1405.

HaLl T.A. 1999: BioEdit: a user-friendly biological sequence alignment editor and analysis program for Windows 95/98/NT. Nucleic Acids Symp. Ser. 41: 95-98.

Heiniger H., Gunter N.L., Adlard R.D. 2008: Relationships between four novel ceratomyxid parasites from the gall bladders of labrid fishes from Heron Island, Australia. Parasitol. Int. 57: $158-165$

Køie M., Karlsbakk E., Nylund A. 2008: The marine herring myxozoan Ceratomyxa auerbachi (Myxozoa: Ceratomyxidae) uses Chone infundibuliformis (Annelida: Polychaeta: Sabellidae) as invertebrate host. Folia Parasitol. 55: 100-104.

Køie M., Whipps C.M., Kent M.L. 2004: Ellipsomyxa gobii (Myxozoa: Ceratomyxidae) in the common goby Pomatoschistus microps (Teleostei: Gobiidae) uses Nereis spp. (Annelida: Polychaeta) as invertebrate hosts. Folia Parasitol. 51: 14-18.

Lom J., Arthur J.R. 1989: A guideline for the preparation of species descriptions in Myxosporea. J. Fish Dis. 12: 151-156.

Meglitsch P.A. 1960: Some coelozoic myxosporidia from New Zealand fishes. I. General and family Ceratomyxidae. Trans. R. Soc. N.Z. 88: 265-365.

Molnár K., Székely C., Hallett S., Atrinson S. 2009: Some remarks on the occurrence, host-specificity and validity of Myxobolus rotundus Nemeczek, 1911 (Myxozoa: Myxosporea) Syst. Parasitol. 72: 71-79.

Received 14 December 2009
Moser M., Kent M.L., Dennis D. 1989: Gall bladder Myxosporea in coral reef fishes from Heron Island, Australia. Aust. J. Zool. 37: 1-13.

Palenzuela O., Redondo M.J., Alvarez-Pellitero P. 2002: Description of Enteromyxum scophthalmi gen. nov., sp. nov. (Myxozoa), an intestinal parasite of turbot (Scophthalmus maximus L.) using morphological and ribosomal RNA sequence data. Parasitology 124: 369-379.

Posada, D., Crandall K.A. 1998: Modeltest: testing the model of DNA substitution. Bioinformatics 14: 817-818.

Ronquist F., Huelsenbeck J.P. 2003: MrBayes 3: Bayesian phylogenetic inference under mixed models. Bioinformatics 19: 1572-1574.

SwOFFord D.L. 2003: PAUP*. Phylogenetic analysis using parsimony (*and other methods). Massachusetts, Sinauer Associates, Sunderland.

Thompson J.D., Higgins D.G., Gibson T.J. 1994: Clustal W: improving the sensitivity of progressive multiple sequence alignment through sequence weighting, position-specific gap penalties and weight matrix choice. Nucleic Acids Res. 22: 4673-4680.

Whipps C.M., Adlard R.D., Bryant M.S., Lester R.J.G., FindLAY V., Kent M.L. 2003: First report of three Kudoa species from Eastern Australia: Kudoa thyrsites from Mahi mahi ( $\mathrm{Co}$ ryphaena hippurus), Kudoa amamiensis and Kudoa minithyrsites $\mathrm{sp}$. n. from Sweeper (Pempheris ypsilychnus). J. Eukaryot. Microbiol. 50: 215-219.

Whipps C.M., Grossel G., Adlard R.D., Yokoyama H., Bryant M.S., Munday B.L., Kent M.L. 2004: Phylogeny of the Multivalvulidae (Myxozoa: Myxosporea) based on comparative ribosomal DNA sequence analysis. J. Parasitol. 90: 618-622.

Accepted 2 March 2010 